\title{
The experimental research of the Polycrystalline Ni-Ti alloy grain deformation under load
}

\author{
Jie Han ${ }^{1, \text { a }}$, Wenyu An ${ }^{2}$, Changbin Yu², Wenguang $\mathrm{Xie}^{2}$, Yan $\mathrm{Li}^{2}$, Zhongjing $\mathrm{Li}^{2}$, and Qiang \\ Wang ${ }^{2, b}$ \\ ${ }^{1}$ College of Information Science and Engineering, Heilongjiang University, China \\ ${ }^{2}$ FCollege of Physics Science and Technology, Heilongjiang University, China \\ a1993013@hlje.edu.cn, bwangqiangid@aliyun.com
}

Keywords: SEM, Digital speckle correlation method (DSCM), Ni-Ti alloy

\begin{abstract}
We propose and demonstrate a technique for monitoring the deformation of the Ni-Ti alloy grain and grain boundary, and analyzing the interaction effects between the grains using DSCM and SEM methods. This work demonstrates that the grain size influences on the mechanical property of the Ni-Ti alloy, grain boundary coordinates the grain interaction during tension.
\end{abstract}

\section{Introduction}

$\mathrm{Ni}$-Ti alloys are shape memory alloy which have be widely applied to various fields. With the micro-machine and information technologies development, Ni-Ti alloys would play a much more significant role in smart materials. Based on the advantages and characteristics of Ni-Ti alloys, understanding the Ni-Ti alloys deformation, the grain boundary and grain interior deformation and grain interaction effects on the micro-scale is important for real applications, which is benefit to further study the materials in micro-scale, and lay the foundation for building micro theoretical models. In this work, the grain and grain boundary deformation figures of the Ni-Ti alloys were recorded under tension using SEM. Combined with DSCM method, the displacement field of the grains during the whole deformation process were obtained. Through the corresponding difference calculate method, the strain field can be got at last. It would be more convenient and exactly for capture the grain deformation and quantified data in micro-scale by combining the SEM and DSCM methods. The results also showed that grain boundary plays a coordinating role in tension process, and the grain size posed to some extent determine the machine properties of the Ni-Ti alloys.

\section{Methods}

\subsection{DSCM and SEM Techniques.}

DSCM has become one of the most intriguing experimental measurement methods among the modern optical measurement mechanics in the last ten years. In some application areas and complex measurement environment, DSCM has its unique advantage. The basic principle of DSCM is introduced as following: (1) Two images of the film's surfaces before and after deformation are captured and compared the difference; (2) The displacement field of film's surface could be measured using interrelation of probability statistics; (3) The calculation formula of deformation could be analyzed and the strain distribution of the film's surface could be calculated. The precision and calculated method of the correlation coefficient plays a key role in DSCM. SEM has many advantages, such as long depth of field, flexible magnification, and high resolution. So SEM may be used to study on properties of materials by many researchers, through combined with DSCM. Peng Rui-dong et al. have investigated strain distributions of various materials through DSCM and SEM.

They attempted and analyzed the effect of the calculation results to the coefficient using DSCM, and the influence secondary electron image and scanning back scattered electron of the SEM. The methods presented in their thesis are verified by comparing the results with those of experimental 
measurements and other software. The calculation results are more accurate and stability, whose coefficient is higher.

The correlation coefficient is match of gray distribution for image sub-region, which could reflect regional similarity between before and after deformation images11. The coefficient is given by the expression (Eq. 1)

$$
C(u, v)=\frac{\sum_{i=1}^{m} \sum_{j=1}^{m}\left[I_{1}(x, y)-\overline{I_{1}}\right]\left[I_{2}\left(x^{\prime}, y^{\prime}\right)-\overline{I_{2}}\right]}{\sqrt{\sum_{i=1}^{m} \sum_{j=1}^{m}\left[I_{1}(x, y)-\overline{I_{1}}\right]^{2}} \sqrt{\sum_{i=1}^{m} \sum_{j=1}^{m}\left[I_{2}\left(x^{\prime}, y^{\prime}\right)-\overline{I_{2}}\right]^{2}}}
$$

Where $I_{1}(x, y)$ and $C(u, v)$ are the functions of gray for before and after deformation images, $I_{1}$ and $\bar{I}_{2}$ are the average of $I_{1}(x, y)$ and $I_{2}\left(x, y^{\prime}\right), C(u, v)$ is correlation. The maximum value of Eq. (1) can be calculated by Newton-iterative method, and then the displacement of sub segment can be given, the strain is derived from by using displacement distribution at last.

\subsection{Experimental system and samples.}

The morphology evolution of the samples during the loading process using real-time control was observed by Shimadzu all-digital hydraulic SEM (as shown in Figure 1). The deformation of the grain and grain boundary were calculated by DSCM. The specimen size is shown in Figure 2, a serious of samples with different grain size and state were tested by a vendor offered parameter. It is clearly seen that different grain pictures of the $\mathrm{Ni}$-Ti alloys samples after polishing and etching in the Figure 3 to 6 . Grain and grain boundaries can be distinguished, which are in accordance with the data in the Table 1.
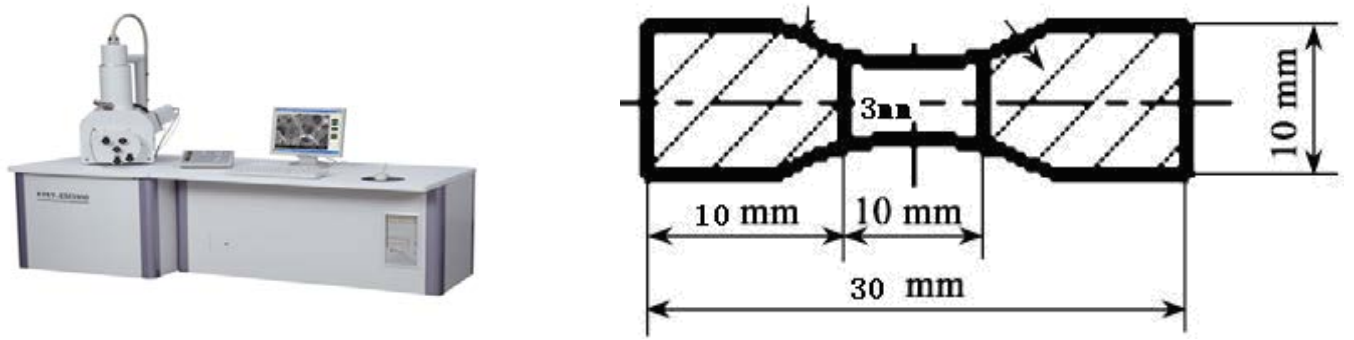

Figure 1. Scanning electron microscopic image. Figure 2. The sizes of the test sample.

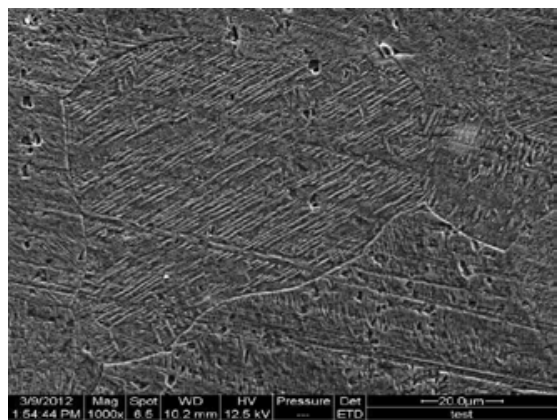

Figure 3. The SEM of grain image of No.1

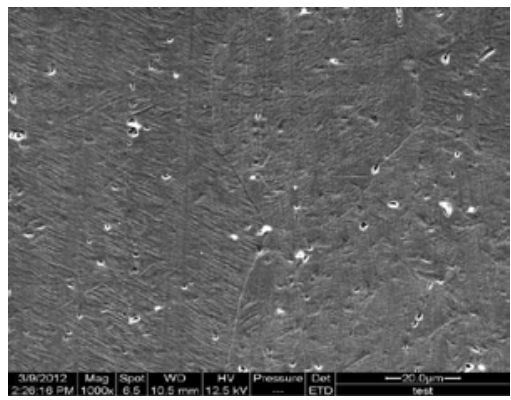

Figure 5. The SEM of grain image of No.4

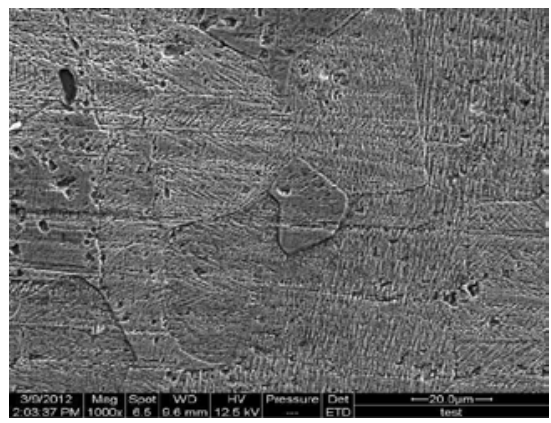

Figure 4. The SEM of grain image of No.2

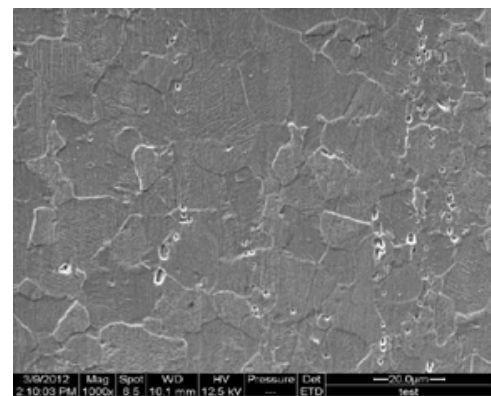

Figure 6 . The SEM of grain image of No.5 
Table 1. The test parameters of the samples

\begin{tabular}{|c|c|c|c|}
\hline Numbers & States & Grain size & $\begin{array}{c}\text { Heat treatment } \\
\text { temperature time }\end{array}$ \\
\hline 1 & Super elastic & $50 \mu \mathrm{m}$ & $700^{\circ} \mathrm{C} / 1 \mathrm{~h}$ \\
\hline 2 & Super elastic & $70 \mu \mathrm{m}$ & $850^{\circ} \mathrm{C} / 1 \mathrm{~h}$ \\
\hline 3 & Super elastic & $40 \mu \mathrm{m}$ & $750^{\circ} \mathrm{C} / \mathrm{h}$ \\
\hline 4 & Super elastic & $60 \mu \mathrm{m}$ & $650^{\circ} \mathrm{C} / 1 \mathrm{~h}$ \\
\hline 5 & Shape memory & $20 \mu \mathrm{m}$ & $500^{\circ} \mathrm{C} / 1 \mathrm{~h}$ \\
\hline
\end{tabular}

\section{Results}

The samples were screwed to the fixtures of the loading system, and under a radial stretch with tensile rate $0.4 \mathrm{~mm} / \mathrm{min}$. Figure 7 shows the relationships of the tensile and the time for the sample No.1, which is similar with other samples tensile curves.

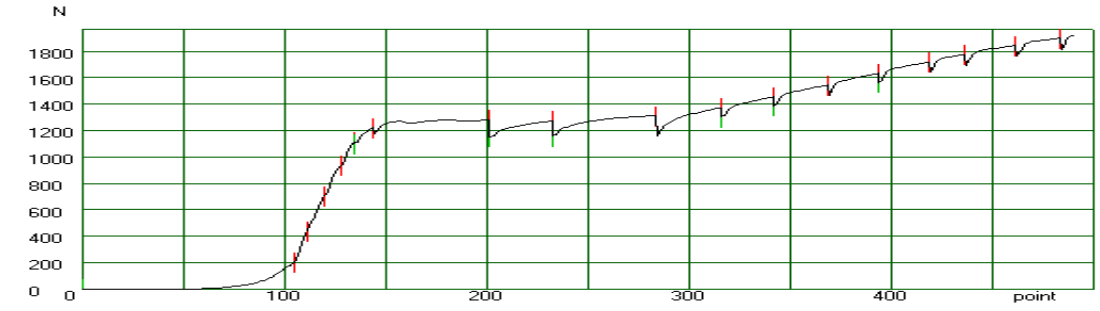

Figure 7. The relation curve between tension and time of NO.1

It is clearly shown that the increasing of the tensile is slower during the first 100s, the sample was pre-tensioned for tension. Between the $200 \mathrm{~N}$ and $1200 \mathrm{~N}$, the tension grows quickly with the time. Whereas, over the $1200 \mathrm{~N}$, the curve of the tension starts to level off, goes on for some time and increases to ultimate strength of the loading system $(2000 \mathrm{~N})$ at last. In the last phase, the tension rise has slowed. Some physical properties of samples presented anisotropic characteristics due to the grains with different crystal orientations. The ratio between the load and cross-sectional area is defined as nominal stress.

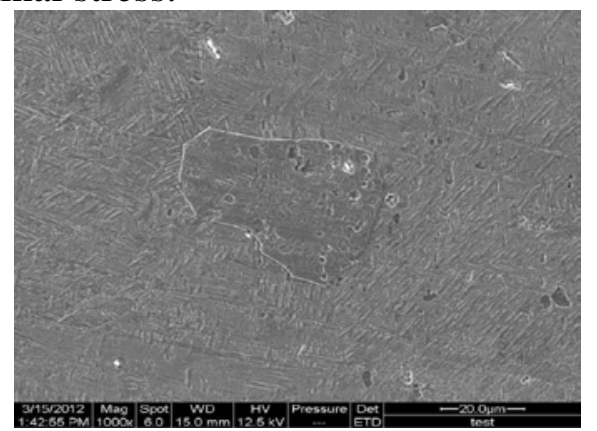

(a) $0 \mathrm{MPa}$

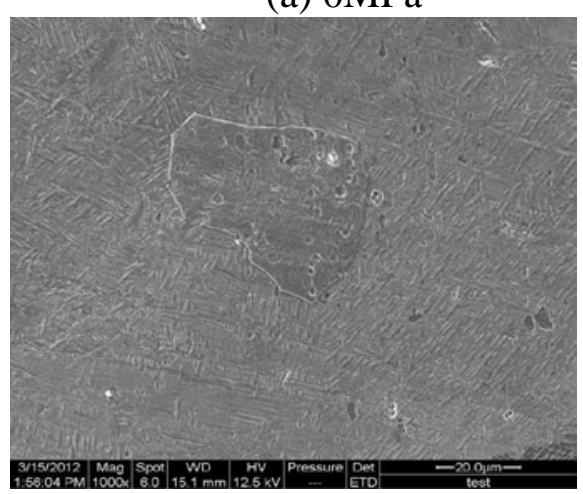

(c) $46 \mathrm{MPa}$

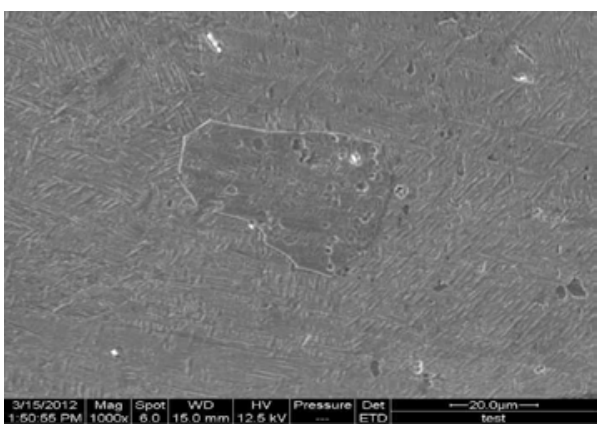

(b) $40 \mathrm{MPa}$

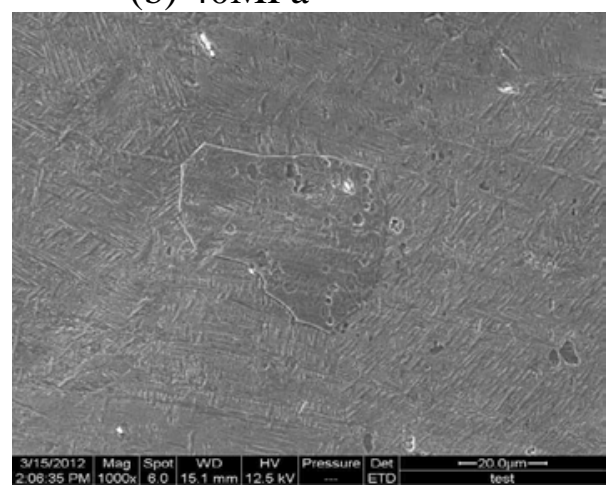

(d) $63 \mathrm{MPa}$

Figure 8 . The record of deformation of the grain 
One grain continuous images of sample No. 1 were tracked with $0 \mathrm{MPa}, 40 \mathrm{MPa}, 46 \mathrm{MPa}$ and 63 MPa separately in the Figure 8. There are obvious deformations on the grain during the tensile process. The grain was stretched in vertical direction and compressed in horizontal direction. For the better description of the deformation characterizes, we worked out the stress distribution across $\mathrm{X}$ and Y directions using DSCM (as shown in Figure 9).

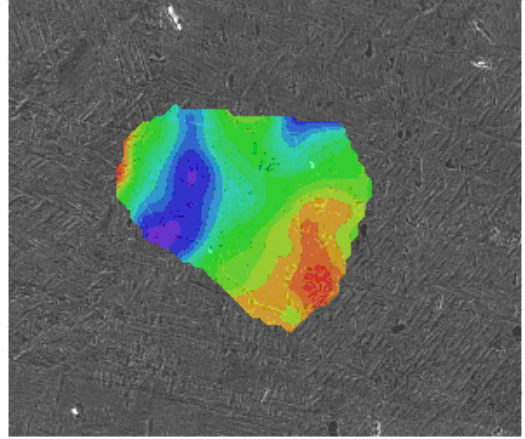

$-0.0056$

(a) 40MPa, $\mathrm{X}$ direction

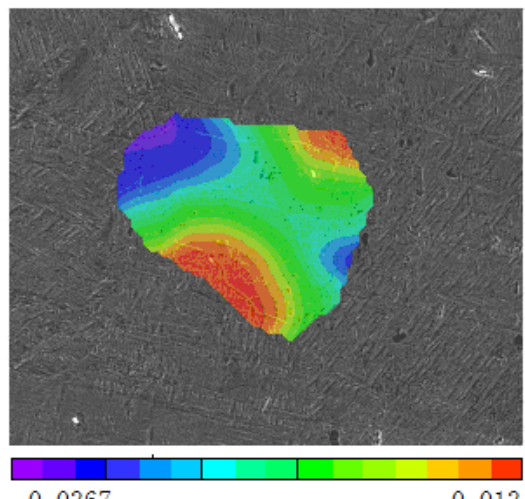

(c) 46MPa, X direction

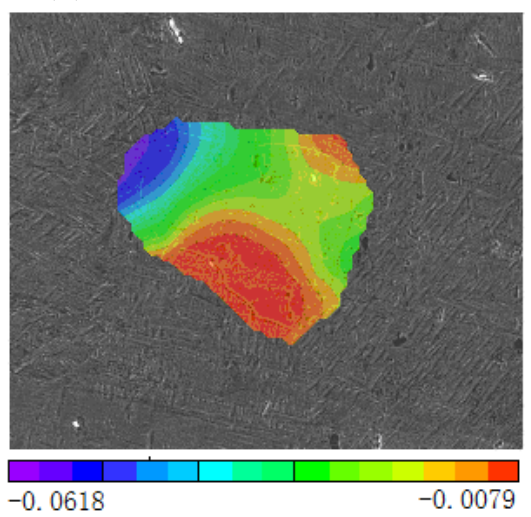

(e) 63MPa, $\mathrm{X}$ direction

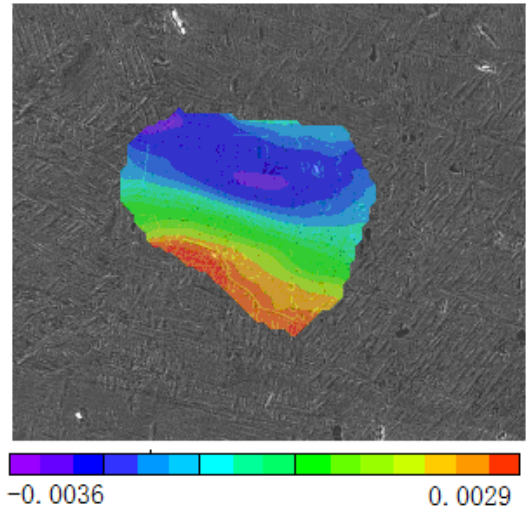

(b) 40MPa, Y direction

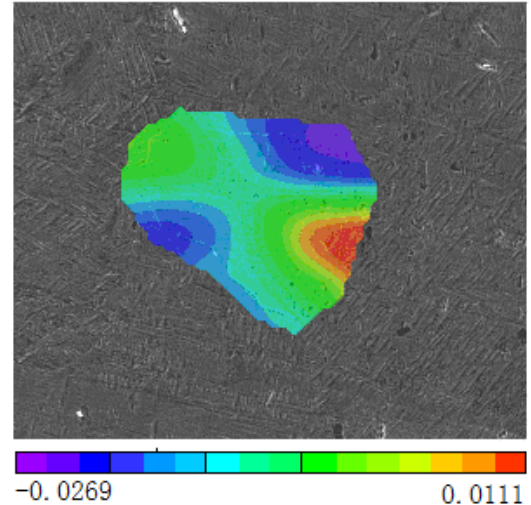

(d) 46MPa, Y direction

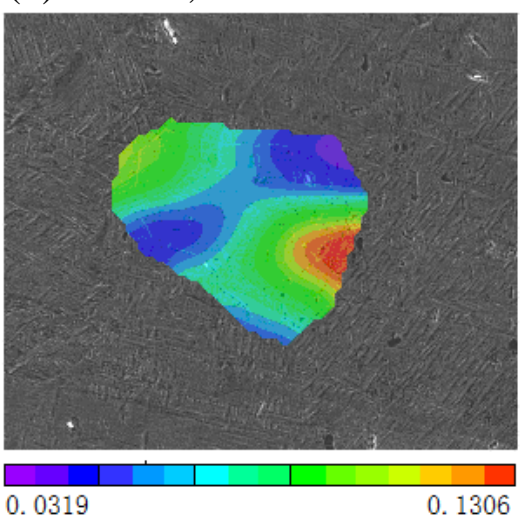

(f) 63MPa, Y direction

Figure 9. The strain of crystalline grain

The relationship of tension and time could be obtained by experiments and computation, which basically showed good agreement. The Ni-Ti alloy specimens were subjected significant tension and exhibited good mechanical performance after the tension reached $200 \mathrm{~N}$ (6.7 MPa). There are no change of the grains and grain boundaries. When the tension reached $1200 \mathrm{~N}-1400 \mathrm{~N}$ (40MPa-43MPa), the specimen became super-elasticity. After some time, the slope of that curve has risen, which is fewer compared with significant changed before. The grains have been great changed--up to $10 \%$ strain changed in some areas. The specimens had not recovered their original shapes after unloading.

There are also significant differences between the NiTi alloy with shape memory effect and super-elasticity alloy. It is seen that the curve leaves obviously rise after applied tension, whereas the slope of the curves suddenly reduced above the $400 \mathrm{~N}(13 \mathrm{MPa})$ at the end of the experiment, with a 
flat regions nowhere in super-elasticity specimen. The specimens had not recovered their original shapes after unloading.

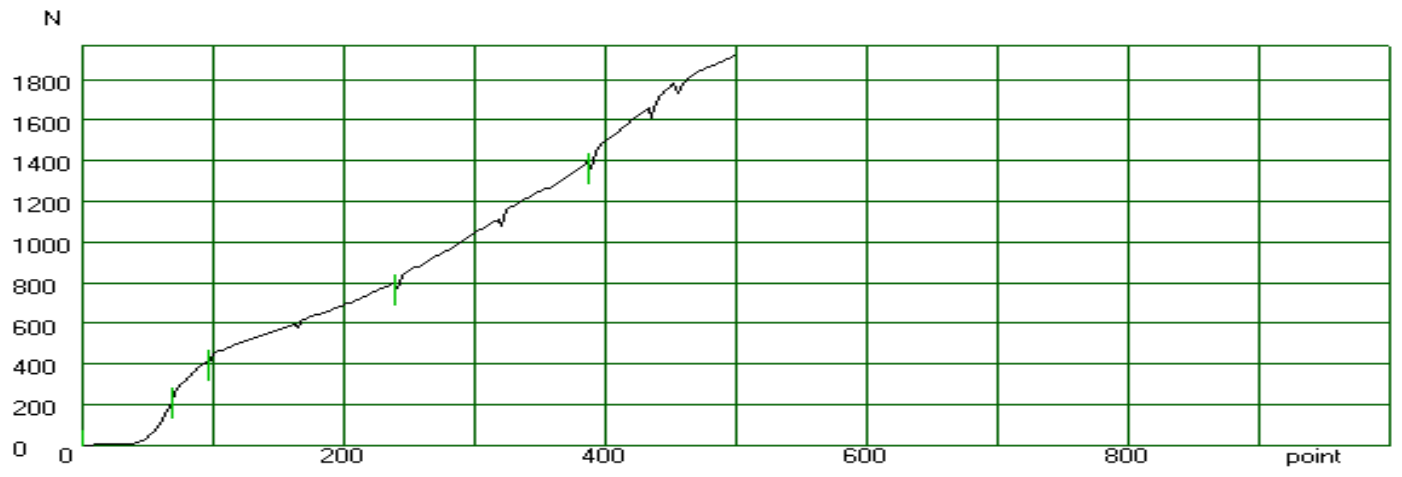

Figure 10. The relation curve between tension and time of NO.5

The deformations of the one grain for every specimen were monitored in real time during our experiment process. Some images were captured with corresponding the different loading, and then a serious strain were calculated by DSCM. The grains were tensioned in radial direction, obvious extensibility was observed in Y direction, corresponding contractility also was seen in X direction. Y direction plays a leading role in the deformation process. The maximum loading for five numbers specimens is about $2000 \mathrm{~N}$. The maximum strains for the grains under the same conditions, are listed in Table 2.

Table 2. The relationship between dimensions and strain

\begin{tabular}{|c|c|c|c|c|c|}
\hline Number & 1 & 2 & 3 & 4 & 5 \\
\hline Grain Size & $50 \mu \mathrm{m}$ & $70 \mu \mathrm{m}$ & $40 \mu \mathrm{m}$ & $60 \mu \mathrm{m}$ & $20 \mu \mathrm{m}$ \\
\hline $\begin{array}{c}\text { Maximum Strain of Y } \\
\text { direction (\%) }\end{array}$ & 13 & 11 & 19 & 10 & 10 \\
\hline $\begin{array}{c}\text { Maximum Strain of X } \\
\text { direction (\%) }\end{array}$ & 6.2 & 4.6 & 6.5 & 3.8 & 6.2 \\
\hline
\end{tabular}

The smaller grain size, the larger is the strain, which is the mechanical response during the tension process. Although the deformation of the grains is large-up to $10 \%$ in some areas, the strain of the grain boundary distributed equally, where is no aberrance. The basic mechanical characteristics of the grains agree with the grain boundaries. The deformation of the grain boundary raised with increasing load, however the strain is smaller than the maximum strain of the grain and around areas. It is demonstrated that the grain boundary play a coordination role among the grains. The results of comparative analysis show that the data variation characteristics of Ni-Ti alloys with shape memory effect are identical to those of super-elasticity specimens in Figures 11-12.

\section{Conclusion}

In this investigation, a new method for analyzing the mechanics characteristics of the polycrystal $\mathrm{Ni}-\mathrm{Ti}$ alloys during the tension process is demonstrated by using SEM and DSCM technologies. The strain changes and distributions of the grains and grain boundary are quantified during the tension process. In the super-elastic condition, the larger grain size, the smaller is the strain under the same loading. In the shape memory and super-elastic condition, the deformation of the grain boundary is smaller than the grain in the $\mathrm{X}$ and $\mathrm{Y}$ directions; the mechanics characteristics of the grain boundary agree with their round grains, which play role in coordination. These attractive physical and operational characteristics are ideally suited for further revealing the deformation mechanism and evolvement rules of the NiTi alloys' micro-structures, providing the references for materials designing and processing(This Project is supported by Heilongjiang Natural Science Foundation A2007-09). 

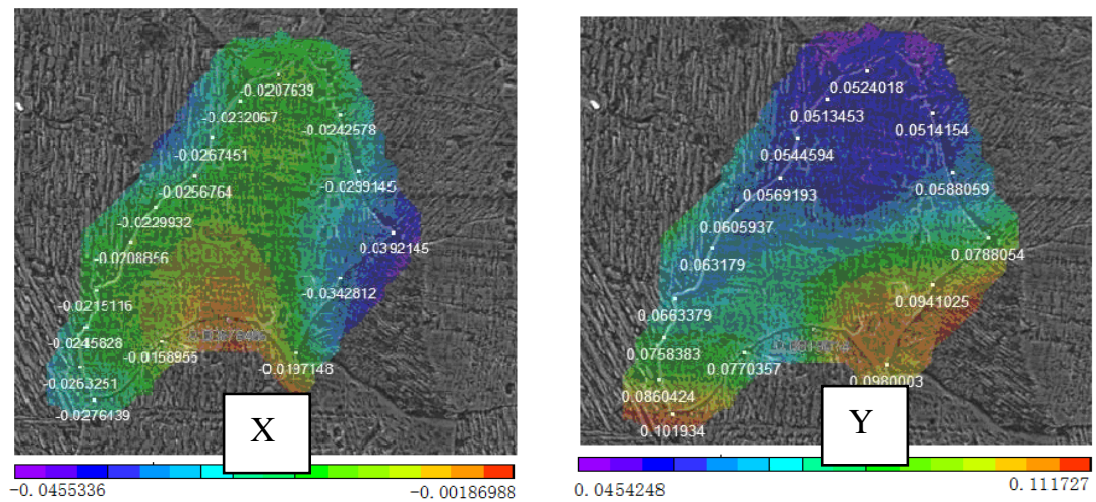

Figure 11. The deformation of the No. 2 grain boundary in $\mathrm{X}$ and $\mathrm{Y}$ directions
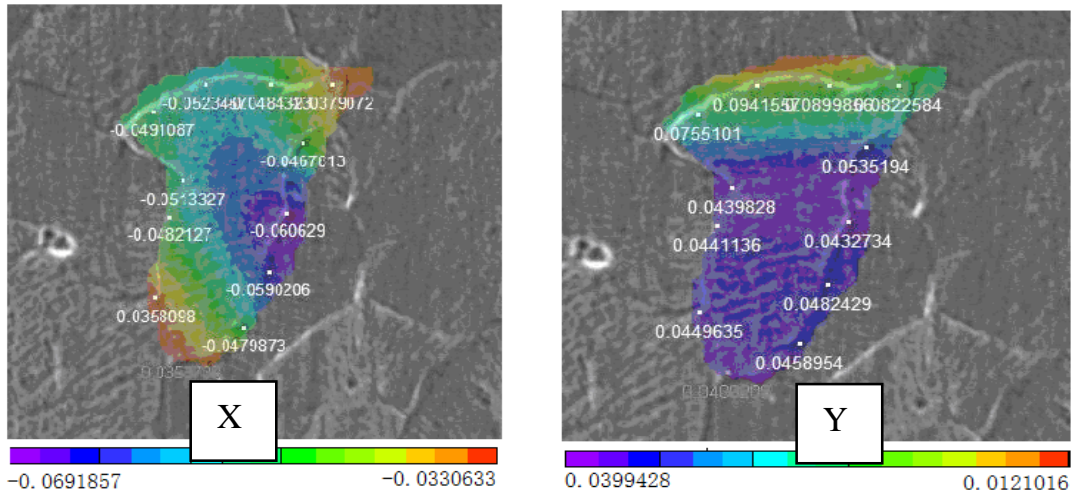

Figure 12. The deformation of the No. 5 grain boundary in $\mathrm{X}$ and $\mathrm{Y}$ directions

\section{References}

[1] K. Otsuka, X. Ren. Physical metallurgy of Ti-Ni-based shape memory alloys. Progress in Materials Science [J]. 2005 (50): 511-678.

[2] Zhu Jian-Xin, Li Yong-Hua2, Meng Fan-Ling, Liu Chang-Sheng. A first principles investigation on NiTi alloy. ACTA PHYSICA SINICA. Vol. 57, No. 11, November, 2008

[3] Wu Xi-jun. Grain Boundary Structure and its Effect on Mechanical Properties of Polycrystalline Materials. Advances in Mechanics [J]. 1990, 20(2):159-173.

[4] Han Tixin, Zeng Xiangguo, Guo Yang, Yang Xin, Chen Huayan, Li Yang. The study of experimental and numerical methods for dynamic fracture process of NiTi alloy. CHINA MEASUREMENT \& TEST.Vol. 42 No.10 October.2016.

[5] Su Y, Zhang Q, Gao Z, et al. Noise-induced bias for convolution-based interpolation in digital image correlation. [J]. Optics Express, 2016, 24(2).

[6] Yong S, Qingchuan Z, Zeren G, et al. Fourier-based interpolation bias prediction in digital image correlation. [J]. Optics Express, 2015, 23(15):19242-60.

[7] Ronghua Z, Huimin X, Zhenxing $\mathrm{H}$, et al. Performances of different subset shapes and control points in subset-based digital image correlation and their applications in boundary deformation measurement. [J]. Applied Optics, 2015, 54(6):1290-301.

[8] Tong W. Reduction of Noise-Induced Bias in Displacement Estimation by Linear Off-Pixel Digital Image Correlation[J]. Strain, 2013, 49(2):158-166.

[9] Pan B. Bias error reduction of digital image correlation using Gaussian pre-filtering[J]. Optics \& Lasers in Engineering, 2013, 51(10):1161-1167.

[10] RUAN Le-le, WANG Wei-ze, Effect of Imaging Mode of Scanning Electron Microscope on the 
results of Digital Speckle Correlation Method. Journal of East China University of Science and Technology[J]. Vol. 41No. 3 2015(6): 412-416.

[11] PENG Rui-dong, WENG Wei, Application of digital speckle correlation method on the measurement of rock deformation by SEM. Journal of China University of Mining \& Technology [J]. Vol. 41 No. 4. Jul.2012:650-656. 